\title{
loMT-based biomedical measurement systems for healthcare monitoring: a review
}

\author{
Imran Ahmed ${ }^{1}$, Eulalia Balestrieri ${ }^{1}$, Francesco Lamonaca ${ }^{2}$ \\ 1 University of Sannio, 82100 Benevento, BN, Italy. \\ 2 University of Calabria, 87036 Arcavacata, Rende (CS), Italy.
}

\begin{abstract}
Biomedical measurement systems (BMS) have provided new solutions for healthcare monitoring and the diagnosis of various chronic diseases. With a growing demand for BMS in the field of medical applications, researchers are focusing on advancing these systems, including Internet of Medical Things (IOMT)-based BMS, with the aim of improving bioprocesses, healthcare systems and technologies for biomedical equipment. This paper presents an overview of recent activities towards the development of loMT-based BMS for various healthcare applications. Different methods and approaches used in the development of these systems are presented and discussed, taking into account some metrological aspects related to the requirement for accuracy, reliability and calibration. The presented loMTbased BMS are applied to healthcare applications concerning, in particular, heart, brain and blood sugar diseases as well as internal body sound and blood pressure measurements. Finally, the paper provides a discussion about the shortcomings and challenges that need to be addressed along with some possible directions for future research activities.
\end{abstract}

Section: RESEARCH PAPER

Keywords: Internet of Things; Internet of Medical Things; loT devices; biomedical devices; biomedical measurement systems; non-invasive medical devices

Citation: Imran Ahmed, Eulalia Balestrieri, Francesco Lamonaca, loMT-based biomedical measurement systems for healthcare monitoring: a review, Acta IMEKO, vol. 10, no. 2, article 24, June 2021, identifier: IMEKO-ACTA-10 (2021)-02-24

Section Editor: Ciro Spataro, University of Palermo, Italy

Received March 6, 2021; In final form April 30, 2021; Published June 2021

Copyright: This is an open-access article distributed under the terms of the Creative Commons Attribution 3.0 License, which permits unrestricted use, distribution, and reproduction in any medium, provided the original author and source are credited.

Corresponding author: Imran Ahmed, e-mail: iahmed@unisannio.it

\section{INTRODUCTION}

Biomedical measurement systems (BMS) play a key role in the detection and diagnosis of various diseases, providing new solutions for healthcare monitoring and improving bioprocesses and technology for biomedical equipment. Generally, BMS use measurement devices to collect vital signs, such as heart rate, pulse rate and body temperature, from the human body, and then these vital signs are processed by a processing unit. Finally, the results are displayed to aid doctors in the diagnosis of various diseases. However, for old-fashioned BMS (for example, nonportable and non-smart ultrasound machines), acquiring vital signs without disturbing the patient's routine activities is challenging. Moreover, these old-fashioned BMS require patients to visit the hospital for their check-up, which takes a great deal of time out of their busy lives. Therefore, in order to utilise BMS without disturbing routine activities, these systems must be able to acquire data in different scenarios [1]. This means not only being limited to situations that require the presence of patients inside hospitals but also outside, for example, industry workers, miners and sports professionals in their working environments as well as military personnel and individuals in their home environment. Hence, the use of BMS for today's lifestyle demands that devices belonging to these systems should be compact, user friendly and comfortable for the wearer, with adequate measurement accuracy even in a harsh or complex environment [1], [3]. As a result of these emerging requirements, recent research activities have been directed towards improving BMS by using the Internet of Things (IoT) [4]-[8] and by creating a new paradigm, the Internet of Medical Things (IoMT) [1], [9]. These IoMT solutions are mainly based on wearable and implantable biomedical measurement devices [12] using different sensors, such as tactile [13], silicon [14], polymer [15] and opticalbased sensors [16], [17] or sensors already integrated into commonly used devices, such as smartphones [3], [18]-[23].

Wearable IoMT BMS typically include devices such as smartwatches, armbands, glasses, smart helmets and digital hearing devices [1], [25]. Today, many wearable devices are smart in the sense that they can locally process signals acquired from sensors and transmit measurement data through the network to other connected devices (on a mobile phone or through hospital 

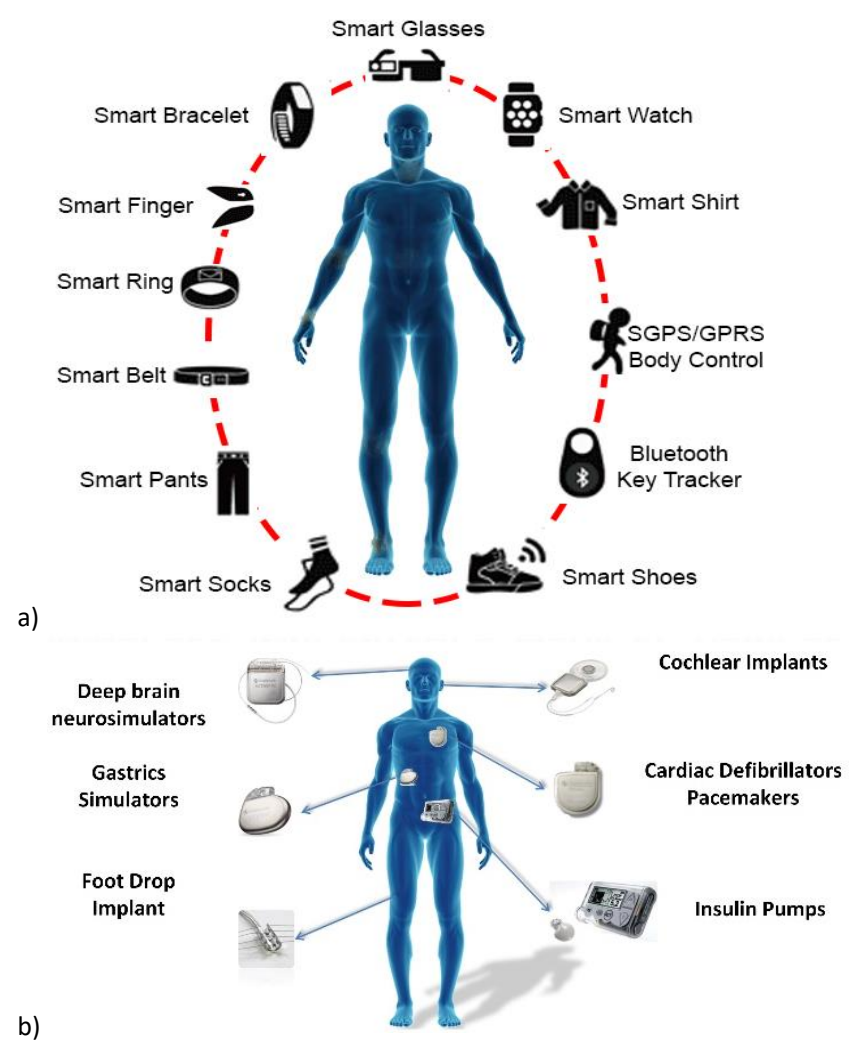

Figure 1. Smart biomedical measurement devices: (a) smart wearable measurement devices [24], (b) smart implantable measurement devices.

systems) so that doctors can promptly monitor and analyse the patient's data to make effective decisions, especially in case of emergency [5], [8].

Figure 1 shows some smart wearable and implantable devices that are used for measuring different vital signs. These devices acquire measurement data and then process and send the data to local elaboration units for further processing and for the presentation of the resulting information to clinicians or patients [12]. Therefore, they are considered a substratum for the development of IoMT BMS. In order to stimulate research for designing innovative BMS, this paper presents an overview of IoMT-based BMS. It is an extended version of a previous contribution to Technical Committee 4 of the 2020 International Measurement Confederation (IMEKO TC4) conference held in Palermo, Italy [9], and takes into account further developments in measurement devices and available techniques used for different medical applications. A discussion concerning each example described is reported, including the advantages, working principles and technology usage. In addition, some general issues and challenges related to the metrological aspects of IoMT-based BMS are highlighted.

The organisation of this paper is as follows. Section II explains the basic architecture of IoMT-based BMS, and Section III presents the five main categories of existing IoMT-based BMS while also introducing some important metrological issues in relation to measurement devices used in IoMT. Section IV discusses the metrological challenges for existing BMS, and finally, the conclusions are presented in Section V.

\section{IOT-BASED BIOMEDICAL MEASUREMENT SYSTEMS (IOMT)}

The main advantage of IoMT-based BMS is that these systems provide the online monitoring of a patient's health to facilitate a quick response in an emergency and to offer remote access to doctors as well as relatives and the patients themselves for monitoring targeted vital signs (blood pressure, heart rate, glucose level and so on) [26]. To this end, IoMT-based BMS are usually designed to offer the following features: (i) the continuous monitoring of parameters without disturbing the patient's daily routine, (ii) an alarm triggered in an emergency, and (iii) the use of low-cost measurement devices. As a consequence, the final aims of an IoMT-based BMS include the following: (i) a reduction in the cost of hospitalisation, (ii) the optimisation of public health costs, (iii) an increase in the independence and quality of life of older adults and (iv) an improvement in the monitoring of hospitalised and/or critical patients.

The general architecture for IoMT-based systems is shown in Figure 2 [27]. Compared with architectures [28]-[30] that are specifically designed for particular applications, such as heart disease, blood pressure and blood sugar, the system shown in Figure 2 is more general and demonstrates the common components belonging to complete IoT-based BMS: (i) a physical layer, (ii) a data integration layer and (iii) an application service/presentation layer.

In the physical layer, IoMT-based BMS mostly use wearable devices to measure the vital signs (heart rate, pulse rate, body temperature, blood pressure, oxygen concentration, lung contraction volume, blood sugar level, respiration rate and so on) of the subjects being monitored. These measurement data are first stored in the storage memory and then transferred to the data integration layer (Figure 2) through the Internet/Bluetooth or any other communication protocol. In the data integration layer, the received data are processed and then sent to the application service/presentation layer. Nowadays, various types of software are available to extract useful information from the measurement data. At the application service/presentation layer, data are analysed by the doctor or experts, enabling them to take effective decisions about the disease. In the following sections, some recently developed IoMT-based systems applied to the diagnosis of various diseases are discussed along with some metrology-related issues.

\section{EXISTING IOMT-BMS CLASSIFICATION OVERVIEW}

IoMT BMS have various medical applications, including healthcare monitoring and the diagnosis of various diseases. Based on these applications, this section has classified the existing IoMT BMS into five main categories: (i) IoMT BMS for heart disease, (ii) IoMT BMS for examining internal body

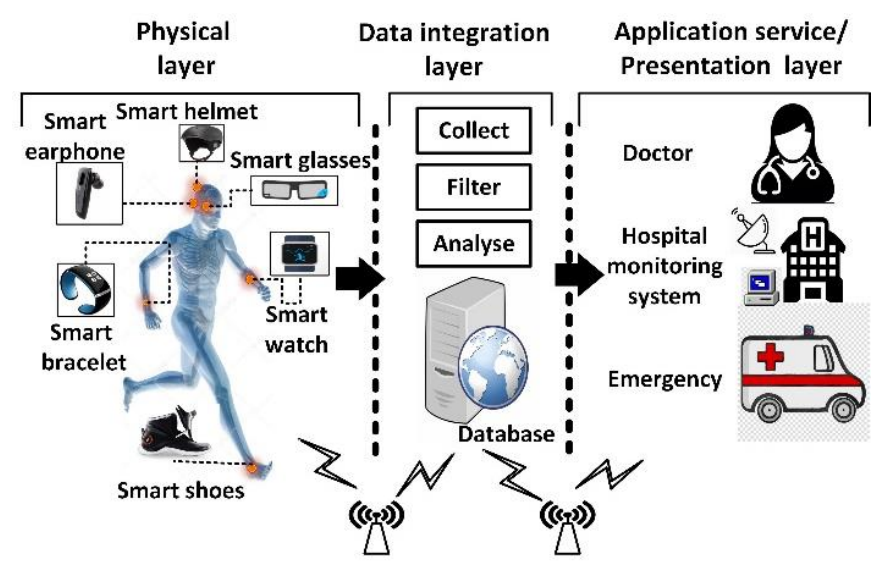

Figure 2. General architecture of IOMT systems. 
sounds, (iii) IoMT BMS for blood pressure, (iv) IoMT BMS for brain diseases and (v) IoMT BMS for blood sugar disease. For each category, various examples are discussed, examining their advantages, working principles and technology usage as well as their reliability and accuracy.

In the literature, it has been observed that researchers in the field of IoMT BMS are usually concerned with applying IoT technologies to different medical applications without focusing on developing suitable solutions in terms of metrology and requirements.

Since IoMT BMS rely on various types of measurement to acquire information about vital signs of the human body, therefore the reliability and accuracy of these systems play a critical and essential role in their actual ability to provide correct and suitable information that can be used by doctors for their diagnoses.

It is also essential that IoMT-BMS devices are properly calibrated to ensure that they are accurate and perform in an appropriate and timely manner. Vital-sign measurements that do not have the required accuracy can result in the misdiagnosis of patients' diseases. Accurate and reliable measurements not only ensure effective treatment but also save time and costs related to misdiagnosed patients.

Thus, the metrological characterisation and calibration of devices are very important for validating the reliability of IoMT systems, and researchers must consider these characteristics, starting at the initial design phase and continuing to the final testing phase of the IoMT BMS. The following subsections discuss existing examples of IoMT BMS and related metrological issues.

\subsection{IoMT BMS for heart disease}

The early detection of heart disease is very important for saving lives, and IoMT could play a vital role in achieving this goal.

In the physical layer, IoMT-based systems for heart-disease detection generally take numerous measurements, such as sugar concentration levels, cholesterol levels, heart rate and pulse rate as well as other vital signs, using sensors. These measurements are usually taken by IoMT devices, such as smartwatches, electrocardiogram (ECG) monitors and other ECG or opticalsensor based heart-monitoring medical devices. Smartwatches mostly use optical sensors to scan the blood flow near the wrist to measure these vital signs, while ECG monitors use electrodes to acquire the electrical signals moving through the heart, record the strength and the timing of these signals and then display the acquired measurements in graphical form. However, there are few limitations to measure these vital signs due to the measurement conditions (for example, a patient sweating during the ECG measurement) and the accuracy of the measuring device [31]. Once the measurements are taken, they are sent to the data integration layer through the Internet and may use cloud-based servers for further processing [32]. After processing, the results are analysed by doctors in the application service/presentation layer by means of a mobile app or web page. Additionally, further algorithms based on artificial intelligence (AI) are now available and are integrated into the data integration layer in order to further aid doctors in the diagnostic process [28]. For example, in [29], an IoMT-based detection system for the monitoring of heart-related diseases based on the deep belief network model and a higher order Boltzmann machine is presented. This system uses Io'T devices, such as embedded sensors and a wearable watch, to measure vital signs, such as heart rate and blood pressure, and to record other physical activities. However, the authors do not provide any details on the types of sensor and wearable watch used in the collection of these data or how accurate these measurements are. After collection, the required data is transmitted to the healthcare centre to be processed using the higher order Boltzmann deep belief neural network (HOBDBNN), which learns the features of heart disease from previous analyses. To evaluate the diseaseprediction accuracy, the system is implemented using MATLAB, and the collected data is divided into two sets: $70 \%$ of the total data is for training the network and $30 \%$ for testing purposes. The HOBDBNN performance is evaluated by different metrics, such as the sensitivity and specificity, Precision and F-measure, [29] which are defined as

$$
\begin{aligned}
& \text { Sensitivity } / \text { Recall }=\frac{\text { True positive }}{\text { True positive }+ \text { False negative }}, \\
& \text { Specificity }=\frac{\text { True negative }}{\text { True negative }+ \text { False positive }}, \\
& \text { Precision }=\frac{\text { True positive }}{\text { True negative }+ \text { False positive }}, \\
& \text { F measure }=2 \times \frac{\text { Precision } \times \text { Recall }}{\text { Precision }+ \text { Recall' }}
\end{aligned}
$$

where True positive is the model's outcome that shows correct prediction of positive class, True negative refers to the correct prediction of a negative class, False positive is the model's outcome that shows incorrect prediction of positive class and False negative is the incorrect prediction of a negative class. These HOBDBNN performance metrics are then compared with other optimised classifiers, such as genetic algorithm-based trained recurrent fuzzy neural networks, swarm-optimised convolution neural networks with a support vector algorithm, particle-optimised feed-forward back-propagated neural networks and particle swarm-optimised radial-basis function networks. The results demonstrate that the performance metrics of the proposed HOBDBNN have better values than those achieved using the other above-mentioned methods. The overall prediction rate of the deep network of the proposed system is reported to be about $99.03 \%$.

Similarly, an ECG-based heart-disease recognition system is presented in [33]. This system measures the heart data by using a commercially available device called the Pulse Sensor AMPED, which consists of a simple optical heart-rate sensor with amplification and noise cancellation hardware components to collect noise-free heart-pulse readings. The collected data are then transmitted wirelessly to the mobile application via an Arduino microcontroller. A monitoring algorithm is implemented in a mobile application to detect any variances from the normal heart rate. This mobile application also raises the alarm whenever an emergency occurs. The system is capable of predicting heart disease by using an intelligent classifier and a machine-learning algorithm, which are pre-trained using clinical data. The authors have reported a $100 \%$ detection rate for monitoring the algorithm and an $85 \%$ correct-classification rate for the classifier.

In [34], an IoMT-based low-power cardiovascular healthcare system with cross-layer optimisation from a sensing patch to a cloud platform is presented. It uses a wearable ECG patch with custom system-on-chip technology that is integrated with a wireless connectivity to connect with mobile devices and a cloud 
platform. To measure and process the ECG signals, the sensing patch needs to be placed directly on the human body. The system performance is evaluated by first checking the signal denoising and compression capability and then by evaluating the correct disease prediction rate using the mobile device and the cloud platform.

To evaluate the signal denoising and compression capability of the proposed system, it is tested on an MIT-BIH database (an open-source dataset that provides standard investigation material for the detection of heart arrhythmia [35]). In particular, various types of noise (a baseline drift noise, power-line noise and electromyography noise) are added to the dataset signals, and, under three different scenarios, the proposed system is evaluated: (i) signal denoising, (ii) signal compression and (iii) combined signal denoising and compression. In this context, some metrics are used, such as the denoised mean square error (MSE), the denoised signal-to-noise ratio $(\mathrm{SNR}, \mathrm{dB})$, improvement in the MSE in percentage, the percentage root mean square difference (PRD), the signal compression ratio (CR) and the quality score (QS), which are defined as

$$
\begin{aligned}
& M S E=\sum_{1}^{N_{i}}\left[f_{c}(n)-f_{r}(n)\right]^{2} / N_{i}, \\
& S N R=10 \log \left[\frac{\sum_{1}^{N_{i}} f_{c}^{2}(n)}{\sum_{1}^{N_{i}}\left[f_{c}(n)-f_{r}(n)\right]^{2}}\right], \\
& P R D=\sqrt{\sum_{1}^{N_{i}}\left[f_{i}(n)-f_{r}(n)\right]^{2},}
\end{aligned}
$$

where $n$ is the number of samples of a signal, $f c(n)$ is the noiseless reference signal, $f r(n)$ is the reconstructed signal after denoising, $f i(n)$ is the input signal and $N i$ is the total length of the input signal.

$$
\begin{aligned}
& C R=N_{i} / M_{\delta}, \\
& Q S=C R / P R D,
\end{aligned}
$$

where $M_{\delta}$ is the number of resolved coefficients after compression.

In the case of signal denoising, the results show that the average improvement in SNR is $12.63 \mathrm{~dB}$ and the improvement in MSE is $94.47 \%$. For signal compression, the results show that the average $\mathrm{CR}$ is 7.89 , the average PRD is $0.61 \%$ and the average QS is 13.06. With regard to combined signal denoising and compression, the average improvement in MSE and SNR is $94.47 \%$ and $12.63 \mathrm{~dB}$, respectively, and the average CR, PRD and QS are 9.8, 6.14\% and 1.62, respectively. In this case, compressed and denoised signals are directly generated with only one iteration of the whole system, which can improve the system efficiency at the cost of sacrificing signal performance (PRD increases and QS decreases). To test the disease-prediction accuracy executed on the cloud platform and mobile device, four kinds of ECG signal are analysed to detect arrhythmias in disease [36]: (i) a normal ECG signal, (ii) a left bundle-branch block (LBBB), (iii) a right bundle-branch block (RBBB) and (iv) paced beats (PB). The five-fold cross validation [36] results for classifying these four kinds of ECG signal, using the mobile device and the cloud platform, are presented. For a normal ECG, the correct classification rate is $96 \%$, for LBBB it is $98 \%$, for $\mathrm{RBBB}$ it is $100 \%$ and for $\mathrm{PB}$ it is $94 \%$. The average correct classification rate of the proposed disease-prediction system executed on the cloud platform is $97 \%$, which is calculated using the mean average correct detection values of all five-fold results [36].

\subsection{IoMT BMS for internal body sounds}

Auscultation is a process for examining internal body sounds, such as from the heart, lungs or other organs, for medical diagnoses. Typically, a stethoscope is used to examine these sounds [46], which can help to detect abnormalities that occur in the human body and provide information about various diseases [46]. However, the auscultation process has some important limitations: it requires the doctor to have good hearing acuity and expertise in order to accurately detect abnormal sounds, the improper placement of the stethoscope on the body results in the improper acquisition of sound, the patient should be in a relaxed position for correct sound acquisition and the presence of background noise can affect the process. In this regard, AIbased auscultation systems have been proved to be very helpful for professionals/doctors in determining abnormal sounds [47], [48]. However, IoT-based auscultation systems allow doctors to remotely monitor their patients and record the sounds, and they offer features for sharing information with other professionals to obtain an immediate second opinion [49]. In this paper, some recently developed IoMT devices related to the measurement of internal body sounds are discussed. For example, eKuore Pro [37] is an IoT-based wireless stethoscope that acquires and measures internal body sounds through devices placed on the body. It can be connected to a mobile app using Wi-Fi to show phonograms in real time and keep track of the patient's medical history, which can be easily shared with professionals and doctors. However, the manufacturer has not provided any information on the accuracy of the system. Another IoMT device for the measurement of internal body sounds currently available on the market, Stethee, is presented in [38]. It is an AI-based wireless stethoscope that uses Aida technology (an AI- and machine-learning-based solution to analyse data [39]) to analyse the heartbeat and lung sounds acquired by placing the device on the body. It uses the Stethee app, which displays clinical information such as heart rate, average systole, average diastole and respiratory rate in only 20 seconds, and it also allows the live streaming of sound data so that it can be visualised in real time for the easy evaluation of vital signs. Although the manufacturer claims that the device has a number of powerful features, but information about measurement methods for the calculation of parameters and their accuracy is not provided. Similarly, the IoMT-based HD Steth system [40] has been introduced by the HD Medical Group for cardiac auscultation. HD Steth is a medical stethoscope approved by the Food and Drug Administration (FDA), and it is composed of integrated ECG electrodes, four microprocessors, an AI-enabled detection system for detecting cardiovascular disease and a display screen to visualise phonocardiographs and electrocardiographs. It provides the real-time visualisation of cardiac waveforms via the Bluetooth Low Energy (BLE) mobile app, and patients can easily share data with specialists via a cloud platform for a remote diagnosis. This device has been patented with noise cancellation and smart amplification for high-fidelity auscultation. As with the previously mentioned devices, the manufacturer has not provided any information on measurement accuracy. Another IoT-based smart stethoscope, StethoMe [41], is a CE certified and intelligent medical device able to measure and classify abnormal sounds in the respiratory system, and it can remotely analyse other internal sounds in the body. StethoMe is able to connect to a smartphone via Bluetooth, and the patient can place 
the device on the points indicated by the mobile app for the recording to start automatically. The mobile app then stores the medical history in the cloud, notifying patients if there are any abnormal sounds, and sends the examination results to a doctor, who will take effective decisions accordingly. It uses AI algorithms to verify the examination process, which makes, in the manufacturer's opinion, the StethoMe about $29 \%$ more accurate than a specialist in the detection and classification of abnormal sounds. However, the accuracy evaluation process is not provided by the manufacturer, so there is no information on how the $29 \%$ figure has been obtained. In [42], an IoMT-based wireless digital stethoscope with mobile integration for sound auscultation is presented. The system first acquires the sound signals from the human body by using a traditional stethoscope chest piece that has an integrated microcontroller unit and a Bluetooth communication device. The acquired data are then processed and finally transmitted to a mobile device for recording and listening to and for the visual display of sound waveforms. This system can be used for monitoring patients in remote locations, especially in quarantine units, and can also be utilised for remotely training healthcare staff through the broadcasting of the recorded signals. However, the authors have not provided any information on the measurement accuracy of the system. In [43], an IoMT-based novel cardiac auscultation monitoring system based on wireless sensing for healthcare is presented. In this system, the cardiac-sound auscultation sensing unit consists of two main components. The first is the HKY-06B heart-sound sensor, manufactured by Huake electronic, which converts the weak heart vibration signal into electrical signals. It also has integrated micro-sound components made from polymer materials. It has the capability of detecting all kinds of heart and acoustic sounds from the body's surface. The second component is the data acquisition module consisting of CC2540 system-on-chip technology with an external antenna [44], an 8051-based micro-controller [45] and other auxiliary components. This module's main function is analogue-to-digital conversion and Bluetooth transmission. It uses a BLE Bluetooth protocol to offer power efficiency and moderate the data transmission rate. The proposed system is used to monitor cardiovascular health, and the acquired information is sent to caregivers as well as medical practitioners using the IoT network and an Android mobile app. In particular, pre-processing, segmentation and clustering techniques are performed to gather any significant health information. The system also features a Hilbert-Huang transform to reduce interference signals and help extract features of the first heart sound, S1, and the second heart sound, S2. In healthy people, S1 and S2 are produced by the closure of the atrioventricular valves and semilunar valves, respectively. The detection rate of the proposed system for S1 and S2 is $88.4 \%$ and $82.7 \%$, respectively, and the overall detection rate of S1 and S2 for irregular heart sounds is $86.66 \%$, as reported in the article. In [46], an IoMT-based smartphone auscultation system is presented. It is a low-cost stethoscope connected to a mobile phone that can record lung sounds and detect abnormal sounds from recorded data. The system uses a support vector machine to identify the sound of wheezes and crackles by extracting features from the spectrogram of each sound signal. The system is trained using recorded data consisting of lung sounds from 155 patients suffering from wheezes or crackles. The system is validated by evaluating the performance of detection algorithms, taking into account the area-under-the-curve (AUC) parameters. This AUC is calculated by plotting the receiver-operating-characteristic (ROC) curve
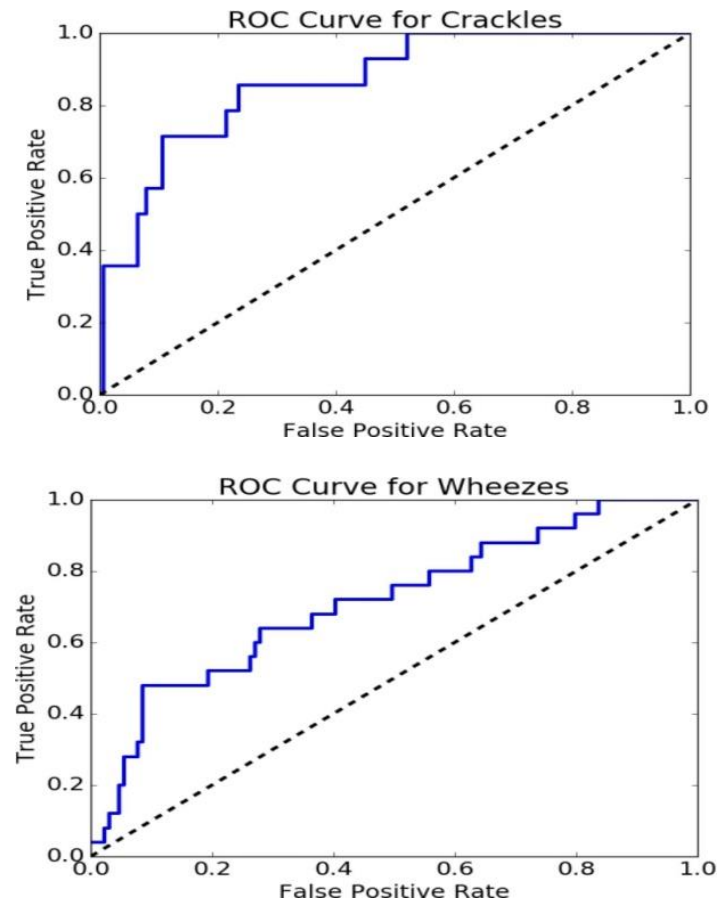

Figure 3. Receiver operating characteristic (ROC) curves for crackles and wheezes [46].

between the false positive rate and the true positive rate, as shown in Figure 3. For the crackle detector algorithm, the AUC value is 0.87 , and for the wheeze algorithm, the AUC value is 0.71. In [49], the IoT-based smartphone monitoring of a second heart sound split is presented. The heart sounds are recorded using a customised external microphone consisting of an acoustic stethoscope and a 3.5-mm mini-plug condenser microphone with adapter that connects wirelessly to a mobile app to record the heartbeat. The system detects S1 and S2 by converting the recorded heartbeat signal into a frequency domain using the fast Fourier transform. S2 is then fed to a discrete wavelet transform (DWT) and a continuous wavelet transform to extract the aortic and pulmonic components. This system can be very useful for remotely monitoring S2. However, these authors have also not provided any information on the measurement accuracy of the system.

\subsection{IoMT BMS for blood pressure}

High blood pressure (BP) is a serious issue that affects older people as well as young adults; it is important for patients to control their BP with repeated check-ups, otherwise serious conditions such as heart failure or strokes can occur. Therefore, patients that suffer hypertension need a BP check-up several times a day. IoMT-based BP measurement systems can help to make this task easier for the patients. An automatic BP measurement system using the oscillometric technique is presented in [50]. This system is capable of monitoring both systolic and diastolic pressure, which are used to define arterial BP. The values are continuously updated through Wi-Fi on a database that can be accessed remotely, where these data are compared with already existing data to improve the accuracy of the results. The authors have stated the accuracy of the system to be $7 \mathrm{mmHg}$. This accuracy has been calculated by means of standards or protocols (defined for BP measuring devices) that are based on the general consensus of several organisations, such as the US Association for the Advancement of Medical 
Instrumentation (AAMI), the British Hypertension Society and the European Society of Hypertension (ESH), which are active working groups on BP monitoring, as well as the International Organization for Standardization (ISO) [51]. However, these protocols and the accuracy of the oscillometric BP instrument continue to be the subject of discussion in the scientific field. This is due, for example, to the BP oscillometric device's tendency to provide inaccurate measurements for certain patient groups and to be prone to noise and artifacts. Moreover, the difficulty in reproducibility of the adopted calibrating methods [52]-[54] allows monitors to pass validation tests when there are clinically significant differences in BP estimated values in some individuals [52].

In [55], the Qardio Arm system is used to develop a smart BP measurement system, in which the acquired oscillometric data are transferred to a smartphone app for analysis and visualisation. The accuracy of the system has been evaluated by comparing its results with the OMRON M3 device, as it has been clinically validated according to the existing ESH International Protocol. However, the same concerns about the accuracy and calibration of the oscillometric BP measurement expressed for the previous device exist for the Qardio Arm system.

In [56], the OMRON HeartGuide is introduced. It is an FDAapproved IoMT smartwatch for BP measurement. This device can measure BP by using an inflatable cuff within the smartwatch bracelet. The smartwatch sends the data to the data integration layer via the Internet, and then sends it on to the application service/presentation layer, where it is available for the doctor to access it in real time. The measurement accuracy of the device is about $3 \mathrm{mmHg}$, but the validation of this device has also been carried out under the protocols for BP devices, with the same limitations reported above.

Similarly, the IoMT-based Instant Blood Pressure (IBP) Auralife app is presented in [57] for BP measurement using a mobile phone and without the use of any external hardware. IBP Auralife extracts the BP values from the photoplethysmogram (PPG) signal acquired with a flash led light and a mobile camera. The accuracy of the system is evaluated by using the AAMI/ISO 81060-2:2013 protocol to compare IBP with other reference upper-arm cuffless BP monitors and oscillometric blood pressure cuff devices. A result analysis shows that the device's systolic BP mean accuracy is $2.7 \mathrm{mmHg}$ and diastolic BP mean accuracy is $2.6 \mathrm{mmHg}$. However, the system only delivers results at this level of accuracy for individuals whose BP lies in a specific range; therefore, it is not suitable for use by individuals whose BP falls outside of the systolic range of $83-178 \mathrm{mmHg}$ or diastolic range of $58-107 \mathrm{mmHg}$. The manufacturer states that it is not recommended for medical use and not a substitute for cuff-based or other BP monitors because it provides an estimate of BP only. Another device, the Asus VivoWatch BP, is reported in [58]. This device has an ECG sensor on the back that receives an ECG signal from the wrist and an optical sensor on the front for the measurement of PPG signals from the index finger. This data is then automatically processed, and the results are displayed to the user. However, the manufacturer has not provided any information on the accuracy of the device.

\subsection{IoMT BMS for brain disease}

Many people are affected by brain diseases, such as brain tumours, dementia, headaches, brain strokes, chronic pains in the head, Tourette's syndrome, Alzheimer's, Parkinson's and epilepsy. The development of IoMT-based BMS in the field of brain-related diseases is a promising solution for the monitoring of patients and timely detection of such diseases. Typical devices that are used in IoMT-based BMS for brain-related diseases are electroencephalogram (EEG) electrodes, smartwatches, galvanic skin response sensors and cameras. These devices are used to monitor brain-disease patients. For example, by using EEG electrodes, it is possible to measure EEG signals to monitor brain activities, with galvanic skin response sensors, the changes in sweat glands can be measured to monitor stress, and by using cameras, it is possible to monitor the daily physical activities of patient (e.g. neuro-degenerative disease patients). In this context, some IoMT brain-related BMS are used to monitor and measure vital signs (for example stress levels and EEG signals) and can generate an alarm in the case of a crisis.

In [59], an IoMT smart sensor for stress-level detection is presented. This is a novel stress-detection system called iStress. It monitors stress levels by measuring parameters such as the rate of body motion, body temperature and levels of sweat during physical activity using sensors for temperature and humidity and also a three-axis accelerometer. The collected data are then processed using a neural network based on a Mamdani-type fuzzy logic controller, which is based on a fuzzy technique. The proposed system is very efficient in terms of power consumption and allows the real-time remote monitoring of stress levels by transmitting the collected data to the cloud, thus helping to improve the detection of the patient's health status. The system classifies stress into three levels: low, normal and medium. The outputs of the sensors are fed to a fuzzy logic controller designed in MATLAB to detect stress levels. The authors report that this system has a stress detection rate of $97 \%$.

In [60], a high-definition camera is used to analyse the motion of the patients with neuro-degenerative diseases (ND). The system is based on remote video monitoring that measures the quantity and quality of two clinically relevant motor symptoms (impairment in step length and arm-swing angle). The system has been evaluated by mean absolute error (MAE), which gives an indication of how close the measurements are to the ground truth. For this evaluation test, a video of a healthy individual in a walking position is recorded in two scenarios: (i) just walking and (ii) sitting on a chair, followed by standing up and walking. The camera is setup to capture the lateral view for the correct detection of the required ND parameters. A total of 23 valid step lengths and 10 arm-swing angles are recorded in both cases. The ground-truth measurements are marked/annotated using the Kinovea software package, which provides a set of tools to capture, slow down, study, compare, annotate and measure the technical performance of a video [61]. The authors state that the system is able to measure ND parameters with a tolerance ranging from $2 \%$ to $5 \%$.

In [62], an IoT-based system is reported that predicts the Parkinson's brain disorder. The system uses wearable IoT-based deep brain simulation (DBS) to collect patient brain activities and assess the condition of cells to predict brain functionality changes. DBS is a smart device that collects brain data by placing electrodes on individuals to conduct continuous monitoring. By means of an heuristic tubu-optimised sequence modular neural network (HTSMNN), it is possible to predict the changes present in the human brain and its functions. To validate the proposed system, the authors have used the dataset in [63] that contains Parkinson's disease-related information. The performance of the system is analysed using MAE, MSE, precision, recall, classification accuracy (CA) and the AUC, which are defined as 


$$
\begin{aligned}
& M A E=\frac{1}{N} \sum_{1}^{N_{i}} y_{i}-\tilde{y}_{i}, \\
& M S E=\frac{1}{N} \sum_{1}^{N_{i}}\left(y_{i}-\tilde{y}_{i}\right)^{2}, \\
& C A=\frac{T P+T N}{T P+T N+F P+F N},
\end{aligned}
$$

where $N$ is the number of Parkinson's features and $y$ is the actual and $\tilde{y}$ the predicted output; TP is the true positive, $T N$ the true negative, $F N$ the false negative and FP the false positive, while precision and recall have already been defined in equations (2) and (4).

The above system parameters are calculated by first calculating the system deviation to identify the errors present in the Parkinson's disease recognition process. The deviation is computed by considering the difference between the actual and predicted output. The HTSMNN system deviation value is compared with other methods, such as particle-swarm-optimised neural networks, particle-swarm-optimised radial basis neural networks, genetic algorithm-based extreme machine-learning networks and tubu-optimised deep neural networks. After computing the above parameters, it is reported by the authors that the system ensures an MAE equal to 0.284, an MSE equal to 0.273 and a CA equal to $98.07 \%$.

In [64], an IoMT-based BMS is presented using the deep learning approach, called Stress-Lysis, which is used to measure stress levels. The deep learning system is developed and tested with three different datasets containing activities of daily living (ADL) and physical activities. ADL are collected with an accelerometer and humidity and temperature sensors worn on the wrist. A physical-activity monitoring dataset is developed by collecting 18 different activities from nine volunteers wearing three inertial measurement sensors and a heart-rate monitor. The system learns the stress-level parameters obtained by the wrist band, such as skin temperature, heart rate and sweat during physical activity. The authors have conducted the validation of this proposed solution by analysing the collected dataset using Python and Tensorflow. The dataset consists of 2,000 samples, of which 1,334 samples are for training purposes and 667 are for testing. The results are displayed in the form of a loss function that demonstrates that the correct classification rate is in the range of $98.3 \%$ to $99.7 \%$.

In [9], a seizure-detection IoMT system is demonstrated by using a DWT, Hjorth parameters and a K-NN classifier. This system is based on an IoMT device called Neuro-Thing, which is capable of accurately detecting seizure-related diseases. In this method, EEG electrodes are used to acquire EEG signals, which contain information on the physiological state of the brain to understand and monitor brain function. These EEG signals are decomposed in sub-bands using DWT, and then Hjorth parameters are extracted from the decomposed signals, which are classified using the K-NN method. The device is capable of sending the information to the IoT cloud, where it can be accessed by doctors/physicians so that they can take effective decisions about the disease. In order to validate the proposed method, the authors perform system-level simulations, implemented in a Simulink environment, where DWT and the K-NN classifier are developed by user-defined functions. IoMT implementation is done using ThingSpeak, which is an open data platform that enables IoT applications to gather and analyse data in the cloud. In addition, the system is validated by experimental results in which open-source EEG data [11] is utilised to validate the classification capability of the K-NN model by calculating sensitivity and specificity, as defined in equations (1) and (2). The reported results show $100 \%$ classification accuracy for normal vs. ictal EEG and $97.9 \%$ for normal and interictal vs. ictal EEG.

\subsection{IOMT BMS for blood sugar disease}

Diabetic or blood sugar disease occurs when the human body is not able to properly process blood sugar [65]. Generally, blood sugar is measured by determining the concentration of glucose in the blood. Most devices are based on electrochemical technology, which uses electrochemical strips to perform measurements. There are some limitations to obtaining accurate measurements due to the variance in strip manufacturing and the use of old or out-of-date strips. Other limitations arise from environmental factors, such as temperature or altitude (in hilly areas), or from patient factors, such as improper handwashing [66]. Patients suffering from this disease usually require their blood glucose levels to be checked regularly and to manage their diet to keep the effects of this disease under control. Recent research focuses on using IoMT to improve the sharing of measurement data with physicians and then giving prompt feedback to patients.

An IoMT system with a novel framework to measure and monitor glucose levels is presented in [65]. The system is used for remotely powered implantable glucose monitoring, in which the signal, retrieved from the interaction of radio frequency signals with biological tissue, is first characterised and then monitored. A low-power Bluetooth protocol is used for the transmission of measurement data to the user's mobile. However, the authors do not discuss the accuracy of the proposed system. In [67], an IoMT-based system for glucose monitoring is presented. The article presents a non-invasive blood glucose measurement system based on optical detection and an optimised regression model. A system for light absorbance at a wavelength of $940 \mathrm{~nm}$ with a prediction model is designed, and the technique is validated through measurements taken from human fingertips. The evaluation of the method is performed by comparing the achieved results with referenced blood glucose concentrations using an SD-check one-touch glucometer. The results are evaluated by calculating the mean absolute difference, which is found to be about $5.82 \mathrm{mg} / \mathrm{dl}$, while the mean absolute relative difference (MARD) is $5.20 \%$, the average error (AvgE) is $5.14 \%$ and RMSE is $7.50 \mathrm{mg} / \mathrm{dl}$. The test samples of 43 healthy people and diabetic patients are collected for a Clarke error grid analysis, which is used to quantify the clinical accuracy of blood glucose devices with reference values [68]. The overall results show that better measurements have been achieved using the proposed approach than using the non-invasive measurement methods presented in [69]-[71].

In [72], iGLU 2.0 is presented. This is a new IoMT-based wearable device that is used for measuring blood glucose levels. The device uses infrared spectroscopy and IoMT paradigms for the remote access of data by doctors/users. Analysis of the optimised regression model is performed, and the system is validated on healthy, pre-diabetic and diabetic patients. The robust regression models of serum glucose levels are deployed as the mechanism for measurement for this proposed solution. In particular, a total of 50 different samples of capillary glucose and 
37 samples of serum glucose are taken from pre-diabetic, diabetic and healthy people for the testing and analysis of the proposed iGLU 2.0 device. The obtained results are then compared with the reference values for serum glucose obtained from a laboratory. In particular, the reference values for capillary glucose are measured using an invasive glucometer SD check, which is the gold standard for validation purposes. In terms of capillary blood glucose, AvgE is found to be about $6.07 \%$ and MARD is $6.08 \%$, whereas for serum glucose, AvgE is $4.88 \%$ and MARD is $4.86 \%$.

In [73], a reliable Io'T-based embedded healthcare system that uses the Alaris 8100 infusion pump, Keil LPC-1768 board and IoT cloud to monitor diabetic patients is proposed. The infusion pump delivers medical liquid (insulin) to the patient on a timely basis, and the Keil LPC-1768 board is responsible for delivering the control commands and daily patient readings and providing a secure connection layer. The system is capable of storing patient records in the cloud, and a secure hash algorithm and secure socket shell are employed in this system to achieve the reliability components of the proposed scheme. The article reports that the system has a $99.3 \%$ probability of continuing to operate normally. Also the authors claim that the proposed system design is reliable, secure and authentic in relation to security and privacy. However, the metrological aspects of the proposed system are not discussed.

\section{METROLOGICAL IOMT-BMS CHALLENGES}

There are several metrological issues related to IoT-based BMS that should be addressed when considering their large-scale implementation in the healthcare sector.

As reported in the previous sections, several devices have been proposed by researchers in this field, but the majority of researchers do not focus on the device's metrological characterisation, and they provide questionable validation, calibration and/or accuracy parameters.

The treatment and monitoring of diseases are based on measurements provided by the devices used in IoMT BMS. If IoMT BMS are not investigated from a metrological perspective, there can be no certainty about their capacity for providing reliable, accurate, precise and repeatable measurements of vital signs, with the serious risk of delivering incorrect information, leading to the misdiagnosis or incorrect treatment of diseases [73]. Therefore, it is important to consider all aspects relating to the measurement accuracy of the devices (measurement nodes) used in the system. In addition, it is essential that the measurement devices used in IoMT BMS are properly calibrated [75] using suitable reproducible procedures and references. There should be appropriate guidelines for the common user on the calibration process of the IoMT device or properly accredited laboratories that provide these services, either onsite or remotely. In practice, calibrations are usually made by switching the device on and off or zeroing or resetting the device, which is not recommended [73]. Due to the presence of various measurement parameters (i.e. pressure flow, temperature, sound pressure), it is difficult to calibrate these devices. Moreover, one of the major challenges is that there is no general consensus among different laboratories (from different disciplines, such as pressure flow, temperature, sound pressure) on how to regulate calibration traceability on a single platform for the different kinds of measurement system [76].

\section{CONCLUSION}

IoMT BMS play an important role in the diagnosis of diseases, such as abnormal blood pressure, heart attacks, brain tumours, Alzheimer's, Parkinson's and epilepsy as well as in healthcare monitoring, the monitoring of disease progression and biomedical research. The rapid growth of and increasing demand for IoMT BMS that suit the modern lifestyle make it essential for these systems to be accurate, fast, user friendly and comfortable for the wearer and to provide stability and accuracy even in harsh environments. Based on these common requirements, scientists are trying to improve these BMS and develop new solutions. The presented overview aims to stimulate research in this field and offer some highlights of IoT-based BMS for specific diseases. The paper has also highlighted some important challenges related to metrology in IoMT that need to be addressed. Such issues will lay the groundwork for the development of new multidisciplinary approaches to the design of improved IoMT systems and, thus, guarantee the continuous monitoring of human health, delivering accurate and reliable measurements.

\section{REFERENCES}

[1] E. Balestrieri, F. Lamonaca, I. Tudosa, F. Picariello, D. Luca Carnì, C. Scuro, F. Bonavolontà, V. Spagnuolo, G. Grimaldi, A. Colaprico, An overview on internet of medical things in blood pressure monitoring, Proc. of the IEEE Int. Symp. on Medical Measurements and Applications (MeMeA), Istanbul, Turkey, 25628 June 2019, pp. 1-6.

DOI: $10.1109 / \mathrm{MeMeA} .2019 .8802164$

[2] D. Luca Carnì, V. Spagnuolo, G. Grimaldi, F. Bonavolontà, A. Liccardo, R. S. Lo Moriello, A. Colaprico., A new measurement system to boost the IoMT for the blood pressure monitoring, Proc. of the IEEE Int. Symp. on Measurements \& Networking (M\&N), Catania, Italy, 8-10 July 2019, pp. 1-6.

DOI: $10.1109 /$ IWMN.2019.8805016

[3] D. L. Carni, D. Grimaldi, P. F. Sciammarella, F. Lamonaca, V. Spagnuolo, Setting-up of PPG scaling factors for $\mathrm{SpO} 2 \%$ evaluation by smartphone, Proc. of the IEEE Int. Symp. on Medical Measurements and Applications, Benevento, Italy, 15-18 May 2016, pp. 430-435. DOI: $\underline{10.1109 / \mathrm{MeMeA} .2016 .7533775}$

[4] D. Luca Carnì, D. Grimaldi, F. Lamonaca, L. Nigro, From distributed measurement systems to cyber-physical systems: a design approach, Int. J. of Computing 16 (2017) pp. 66-73.

[5] F. Lamonaca, C. Scuro, D. Grimaldi, R. S. Olivito, P. F. Sciammarella, D. L. Carni, A layered IoT-based architecture for a distributed structural health monitoring system, ACTA IMEKO 8 (2019) pp. 45-52.

DOI: $\underline{10.21014 / \mathrm{acta} \text { imeko.v8i2.640 }}$

[6] E. Balestrieri, P. Daponte, L. D. Vito, F. Picariello, S. Rapuano, I. Tudosa, A Wi-Fi internet-of-things prototype for ECG monitoring by exploiting a novel compressed sensing method, ACTA IMEKO 9 (2020) pp. 38-45.

DOI: $\underline{10.21014 / \mathrm{acta} \text { imeko.v9i2.787 }}$

[7] I. Tudosa, P. Daponte, L. De Vito, S. Rapuano, F. Picariello, F. Lamonaca, A survey of measurement applications based on IoT, Proc. of the Workshop on Metrology for Industry 4.0 and IoT, MetroInd 4.0 and IoT, Brescia, Italy, 16-18 April 2018, pp. 157162. DOI: $\underline{10.1109 / M E T R O I 4.2018 .8428335 ~}$

[8] E. Balestrieri, L. D. Vito, F. Lamonaca, F. Picariello, Research challenges in measurements for Internet of Things systems, ACTA IMEKO 7 (2018) pp. 82-94.

DOI: $\underline{10.21014 / \mathrm{acta} \text { imeko.v7i4.675 }}$

[9] I. Ahmed, F. Lamonaca, Recent developments in IoMT based biomedical measurement systems: a review, IMEKO TC4 2020, Palermo, Italy, 14 - 16 September 2020, pp 23-28. 
[10] M. A. Sayeed, S. P. Mohanty, E. Kougianos, H. Zaveri., A fast and accurate approach for real-time seizure detection in the IoMT, Proc. of the IEEE Int. Smart Cities Conf., Kansas City, USA, 1619 September 2018, pp. 1-5. DOI: $10.1109 /$ ISC2.2018.8656713

[11] R. G. Andrzejak, K. Lehnertz, F. Mormann, C. Rieke, P. David, C. E. Elger, Indications of nonlinear deterministic and finitedimensional structures in time series of brain electrical activity: dependence on recording region and brain state, Phys. Rev. 64 (2001) p. 061907

DOI: $10.1103 /$ PhysRevE.64.061907

[12] H. C. Koydemir, A. Ozcan, Wearable and implantable sensors for biomedical applications, Annual Rev. of Analytical Chemistry, 11 (2018) pp. 127-146.

DOI: $10.1146 /$ annurev-anchem-061417-125956

[13] P. Saccomandi, E. Schena, C. M. Oddo, L. Zollo, S. Silvestri, E. Guglielmelli, Microfabricated tactile sensors for biomedical applications: a review, Biosensors 4 (2014) pp. 422-448. DOI: $10.3390 /$ bios 4040422

[14] Y. Xu, X. Hu, S. Kundu, A. Nag, N. Afsarimanesh, S. Sapra, S. C Mukhopadhyay, T. Han, Silicon-based sensors for biomedical applications: a review, Sensors 19 (2019) p. 2908. DOI: $10.3390 / \mathrm{s} 19132908$

[15] J. Yunas, B. Mulyanti, I. Hamidah, M. Mohd Said, R. E. Pawinanto, W. A. F. Wan Ali, A. Subandi, A. A. Hamzah, R. Latif, B. Yeop Majlis, Polymer-based MEMS electromagnetic actuator for biomedical application: a review, Polymers 12 (2020) p. 1184 DOI: $10.3390 /$ polym12051184

[16] I. Ahmed, U. Zabit, A. Salman, Self-mixing interferometric signal enhancement using generative adversarial network for laser metric sensing applications, IEEE Access 7 (2019) pp. 174641-174650. DOI: $10.1109 /$ ACCESS.2019.2957272

[17] I. Ahmed, U. Zabit., Fast estimation of feedback parameters for a self-mixing interferometric displacement sensor, Proc. of CCODE, Islamabad, Pakistan, 8-9 March 2017, pp. 407-411. DOI: $10.1109 / C-C O D E .2017 .7918966$

[18] D. L. Carnì, D. Grimaldi, A. Nastro, V. Spagnuolo, F. Lamonaca, Blood oxygenation measurement by smartphone, IEEE Instrumentation \& Measurement Magazine 20 (2017) pp. 43-49. DOI: $10.1109 /$ MIM.2017.7951692

[19] Y. Kurylyak, K. Barbe, F. Lamonaca, D. Grimaldi, W. Van Moer, Photoplethysmogram-based Blood pressure evaluation using Kalman filtering and Neural Networks, Proc. of the IEEE Int. Symp. on Medical Measurements and Applications (MeMeA 2013) Gatineau, QC, Canada, 4-5 May 2013 pp. 170-174. DOI: $10.1109 / \mathrm{MeMeA} .2013 .6549729$

[20] F. Lamonaca, K. Barbe, Y. Kurylyak , D. Grimaldi, W. V. Moer, A. Furfaro, V. Spagnuolo, Application of the artificial neural network for blood pressure evaluation with smartphones, Proc. of the IEEE Int. Conf. on Intelligent Data Acquisition and Advanced Computing Systems, Berlin, Germany, 12-14 September 2013, pp. 408-412. DOI: $10.1109 /$ IDAACS.2013.6662717

[21] F. Lamonaca, D. L. Carnì, D. Grimaldi, A. Nastro, M. Riccio, V. Spagnolo, Blood oxygen saturation measurement by smartphone camera, Proc. of the IEEE Int. Symp. on Medical Measurements and Applications (MeMeA 2015), Pisa, Italy, 7 - 9 May 2015, pp. 359-363.

DOI: $10.1109 / \mathrm{MeMeA} .2015 .7145228$

[22] G. Polimeni, A. Scarpino, K. Barbé, F. Lamonaca, D. Grimaldi., Evaluation of the number of PPG harmonics to assess smartphone effectiveness, Proc. of the IEEE Int. Symp. on Medical Measurements and Applications, Lisbon, Portugal, 11-12 June 2014, pp. 433-438.

DOI: $\underline{10.1109 / M e M e A .2014 .6860101}$

[23] F. Lamonaca, Y. Kurylyak, D. Grimaldi, V. Spagnuolo, Reliable pulse rate evaluation by smartphone, Proc. of the IEEE Int. Workshop on Medical Measurements and Applications (MeMeA2012), Budapest, Hungary, 18 - 19 May 2012, pp. 234237.
DOI: $10.1109 / \mathrm{MeMeA} .2012 .6226672$

[24] Heres Arantes Junqueira, Types of wearable technology. Online [Accessed 21 May 2021] https://www.researchgate.net/figure/Different-types-ofwearable-technology fig5 322261039

[25] R. Nayak, L. Wang, R. Padhye, Electronic textiles for military personnel, in: Electronic Textiles: Smart Fabrics and Wearable Technology. T. Dias (editor), Woodhead Publishing, 2015, pp. 239-256.

[26] S. Vishnu, S. R. J. Ramson, R. Jegan, Internet of Medical Things (IoMT) - an overview, Proc. of ICDCS, Coimbatore, India, 5-6 March 2020, pp. 101-104.

DOI: $\underline{10.1109 / \mathrm{ICDCS} 48716.2020 .243558}$

[27] E. Balestrieri, L. De Vito, F. Picariello, I. Tudosa, A novel method for compressed sensing-based sampling of ECG signals in medical-IoT era, Proc. of the IEEE Int. Symp. on Medical Measurements and Applications (MeMeA), Istanbul, Turkey, 2628 June 2019, pp. 1-6. DOI: $10.1109 / \mathrm{MeMeA} .2019 .8802184$

[28] F. Ahmed, An Internet of Things (IoT) application for predicting the quantity of future heart attack patients, Int. J. Comput. Appl. 164 (2017) pp. 36-40. DOI: $10.5120 /$ IJCA2017913773

[29] Z. Al-Makhadmeh, A. Tolba, Utilizing IoT wearable medical device for heart disease prediction using higher order Boltzmann model: a classification approach, Measurement 147 (2019) pp. 1 9. DOI: $10.1016 / \mathrm{j}$.measurement.2019.07.043

[30] G. Bucci, F. Ciancetta, E. Fiorucci, A. Fioravanti, A. Prudenzi, An internet-of-things system based on powerline technology for pulse oximetry measurements, ACTA IMEKO 9 (2020) pp. 114-120. DOI: $10.21014 /$ acta imeko.v9i4.724

[31] E. Balestrieri, P. Daponte, L. D. Vito, F. Picariello, S. Rapuano, Oscillometric blood pressure waveform analysis: challenges and developments, Proc. of the IEEE Int. Symp. on Medical Measurements and Applications (MeMeA), Istanbul, Turkey, 2628 June 2019, pp. 1-6. DOI: $10.1109 / \mathrm{MeMeA} .2019 .8802175$

[32] G. Joyia, A. Farooq, S. Rehman, R. M. Liaqat, Internet of Medical Things (IOMT): applications, benefits and future challenges in healthcare domain, J. Commun. 12 (2017) pp. 240-247. DOI: $10.12720 / \mathrm{jcm} \cdot 12.4 .240-247$

[33] A. F. Otoom, E. E. Abdallah, Y. Kilani, A. Kefaye, M. Ashour, Effective diagnosis and monitoring of heart disease, Int. J. of Software Engineering and Its Applications 9 (2015) pp. 143-156. DOI: $10.14257 /$ ijseia.2015.9.1.12

[34] C. Wang, Y. Qin., H. Jin, I. Kim, J. G. D. Vergara, C. Dong, Y. Jaing, Q. Zhou, J. Li, Z. He, Z. Zou, L. R. Zheng, X. Wu, Y. Wang, A low power cardiovascular healthcare system with cross-layer optimization from sensing patch to cloud platform, IEEE Trans. on Biomedical Circuits and Systems 13 (2019) pp. 314-329.

DOI: $10.1109 /$ TBCAS.2019.2892334

[35] Goldberger, A., Amaral, L., Glass, L., Hausdorff, J., Ivanov, P. C., Mark, R., Stanley, H. E. (2000). PhysioBank, PhysioToolkit, and PhysioNet: Components of a new research resource for complex physiologic signals. Circulation 101 (23), pp. e215-e220. DOI: $10.13026 / \mathrm{C} 2108 \mathrm{~F}$

[36] Jason Brownlee, A Gentle Introduction to k-fold CrossValidation, 23 May 2018. Online [Accessed 28 June 2021] https://machinelearningmastery.com/k-fold-cross-validation/

[37] Ekuore, eKuore Pro electronic stethoscope. Online [Accessed 28 June 2021] https://www.ekuore.com/wireless-stethoscope/

[38] MEDICINE, Stethee Pro. Online [Accessed 28 June 2021] https://www.stethee.com/

[39] TClab, Artificial Intelligence for Document Automation. Online [Accessed 28 June 2021] https://www.tclab.it/en/aida/

[40] HD Medical Group, Intelligent Stethoscope with Integrated ECG. Online [Accessed 28 June 2021] 
https://hdmedicalgroup.com/wpcontent/uploads/2020/11/HD-Steth-Data-Sheet.pdf

[41] StehthoMe. Online [Accessed 28 June 2021] https://stethome.com/

[42] D. K. Degbedzui, M. Tetteh, E. E. Kaufmann, G. A. Mills, Bluetooth-based wireless digital stethoscope with mobile integration, Biomedical Engineering: Applications, Basis and Communications 30 (2018) p. 1850010.

DOI: $10.4015 / \mathrm{S} 1016237218500102$

[43] H. Ren, H. Jin, C. Chen, H. Ghayvat, W. Chen, A novel cardiac auscultation monitoring system based on wireless sensing for healthcare, IEEE J. of Translational Engineering in Health and Medicine 6 (2018) pp. 1-12.

DOI: $10.1109 /$ JTEHM.2018.2847329

[44] Texas Instruments, 2.4-GHz Bluetooth low energy System-onChip. Online [Accessed 28 June 2021] https://www.ti.com/lit/ds/symlink/cc2540.pdf

[45] Farnell, Atmel 8051 Microcontroller Family - Product Selection Guide. Online [Accessed 28 June 2021] http://www.farnell.com/datasheets/46220.pdf

[46] D. Chamberlain, R. Kodgule, Y. Thorat, V. Das, V. Miglani, D. Ganelin, A. Dalal, T. Sahasrabudhe, A. Lanjewar, R. Fletcher Smart phone-based auscultation platform, European Respiratory J. 48 (2016) suppl. 60. DOI: $10.1183 / 13993003$. congress-2016.OA2000

[47] D. G. McNamara, Value and limitations of auscultation in the management of congenital heart disease, Pediatr. Clin. North America 37 (1990) pp. 93-113. DOI: $10.1016 / \mathrm{s} 0031-3955(16) 36834-1$

[48] G. Xavier, C. A. Melo-Silva, C. E. V. G. D. Santos, V. M. Amado, Accuracy of chest auscultation in detecting abnormal respiratory mechanics in the immediate postoperative period after cardiac surgery, J Bras. Pneumol. 45 (2019) pp. 1-8. DOI: $10.1590 / 1806-3713 / \mathrm{e} 20180032$

[49] S. R. Thiyagaraja, J. Vempati, R. Dantu, T. Sarma, S. Dantu, Smart phone monitoring of second heart sound split, Proc. of the 36th Annual Int. Conf. of the IEEE Eng. in Medicine and Biology Society, Chicago, USA, 26-30 August 2014, pp. 2181-2184. DOI: $10.1109 /$ EMBC.2014.6944050

[50] A. Varghese, M. Raghvan, N. S. Hegde, N. T. Prathiba, M. Ananda, IoT based automatic blood pressure system, Int. J. of Science \& Research (2015) pp. 1-3. Online [Accessed 28 June 2021] https://www.ijsr.net/conf/RISE2017/IJSR1.pdf

[51] G. S. Stergiou, B. Alpert, S. Mieke, R. Asmar, N. Atkins, S. Eckert, G. Frick, B. Friedman, T. Graßl, T. Ichikawa, J. P. Ioannidis, P. Lacy, R. McManus, A. Murray, M. Myers, P. Palatini, G. Parati, D Quinn, J. Sarkis, A. Shennan, T. Usuda, J. Wang, C. O. Wu, E. O'Brien, A universal standard for the validation of blood pressure measuring devices, Hypertension 71 (2018) pp. 368-374. DOI: $\underline{\text { 10.1161/HYPERTENSIONAHA.117.10237 }}$

[52] E. Balestrieri, S. Rapuano, Instruments and methods for calibration of oscillometric blood pressure measurement devices, IEEE Trans. on Instrumentation and Measurement 59 (2010) pp. 2391-2404. DOI: $10.1109 /$ TIM.2010.2050978

[53] E. Balestrieri, P. Daponte and S. Rapuano, Automated noninvasive measurement of blood pressure: standardization of calibration procedures, Proc. of the IEEE Int. Workshop on Medical Measurements and Applications, Ottawa, Canada, 9-10 May 2008, pp. 124-128. DOI: $10.1109 /$ MEMEA.2008.4543012

[54] E. Balestrieri, P. Daponte, S. Rapuano, Towards accurate NIBP simulators: manufacturers' and researchers' contributions, Proc. of the IEEE Int. Symp. on Medical Measurements and Applications (MeMeA), Gatineau, Canada, 4-5 May 2013, pp. 91-96. DOI: $10.1109 / \mathrm{MeMeA} .2013 .6549713$

[55] Qardio. [Accessed 28 June 2021] https://www.getqardio.com/
[56] Manualslib, Omron HeartGuide BP8000-M Instruction Manual page 67. Online [Accessed 28 June 2021] https://www.manualslib.com/manual/1501475/OmronHeartguide-Bp8000-M.html?page $=67 \#$ manual

[57] Instant Blood Pressure app. Online [Accessed 28 June 2021] https://www.instantbloodpressure.com/

[58] Asus. Online [Accessed 28 June 2021] https://www.asus.com

[59] L. Rachakonda, P. Sundaravadivel, S. P. Mohanty, E. Kougianos, M. Ganapathiraju, A smart sensor in the IoMT for stress level detection, Proc. of the IEEE Int. Symp. on Smart Electronic Systems (ISES) Hyderabad, India, 17-19 December 2018, pp. 141 145. DOI: $10.1109 /$ iSES.2018.00039

[60] B. Abramiuc, S. Zinger, P. H. N. de With, N. de Vries-Farrouh, M. M. van Gilst, B. Bloem, S. Overeem, Home video monitoring system for neurodegenerative diseases based on commercial HD cameras, Proc. of the 5th ICCE, Berlin, Germany, 6-9 September 2015, pp. 489-492. DOI: $10.1109 /$ ICCE-Berlin.2015.7391318

[61] Kinovea software. Online [Accessed 25 June 2021] https://www.kinovea.org/

[62] A. Ali Al Zubi, A. Alarifi, M. Al-Maitah, Deep brain simulation wearable IoT sensor device based Parkinson brain disorder detection using heuristic tubu optimized sequence modular neural network, Measurement 161 (2020), pp. 107887.

DOI: $10.1016 /$ j.measurement.2020.107887

[63] G. M. Mashrur E. Elahi, S. Kalra, L. Zinman, A. Genge, L. Korngut, Y.-H. Yang. Texture classification of MR images of the brain in ALS using M-CoHOG: a multi-center study, Comput. Med. Imaging Graph. (2020), pp. 101659

DOI: $10.1016 /$ i.compmedimag.2019.101659

[64] L. Rachakonda, S. P. Mohanty, E. Kougianos, P. Sundaravadivel, Stress-Lysis: a DNN-integrated edge device for stress level detection in the IoMT, IEEE Trans. on Consumer Electronics 65 (2019) pp. 474-483. DOI: $10.1109 /$ TCE.2019.2940472

[65] M. Ali, L. Albasha, H. Al-Nashash, A Bluetooth low energy implantable glucose monitoring system, Proc. of the 8th European Radar Conf., Manchester, UK, 12-14 October 2011, pp. 377-380.

[66] B. H. Ginsberg, Factors affecting blood glucose monitoring: sources of errors in measurement, J. of Diabetes Science and Technology 3 (2009) pp. 903-913. DOI: $10.1177 / 193229680900300438$

[67] P. Jain, S. Pancholi, A. M. Joshi, An IoMT based non-invasive precise blood glucose measurement system, Proc. of iSES (Formerly iNiS), Rourkela, India, 16-18 December 2019, pp. 111116. DOI: $10.1109 /$ iSES47678.2019.00034

[68] W. L. Clarke, D. Cox, L. A. Gonder-Frederick, W. Carter, S. L. Pohl, Evaluating clinical accuracy of systems for self-monitoring of blood glucose, Diabetes Care 10 (1987) pp. 622-628. DOI: $\underline{10.2337 / \text { diacare.10.5.622 }}$

[69] K. Song, U. Ha, S. Park, J. Bae, H. Yoo, An impedance and multiwavelength near-infrared spectroscopy IC for non-invasive blood glucose estimation, IEEE J. of Solid-State Circuits 50 (2015), pp. 1025-1037. DOI: $10.1109 /$ JSSC.2014.2384037

[70] N. Demitri, A. M. Zoubir, Measuring blood glucose concentrations in photometric glucometers requiring very small sample volumes, IEEE Trans. on Biomedical Engineering 64 (2017) pp. 28-39

DOI: $10.1109 /$ TBME.2016.2530021

[71] G. Acciaroli, M. Vettoretti, A. Facchinetti, G. Sparacino, C. Cobelli, Reduction of blood glucose measurements to calibrate subcutaneous glucose sensors: a Bayesian multiday framework, IEEE Trans. on Biomedical Engineering 65 (2018) pp. 587-595 DOI: $10.1109 /$ TBME.2017.2706974

[72] A. M. Joshi, P. Jain, S. P. Mohanty, N. Agrawal, A new wearable for accurate non-invasive continuous serum glucose measurement 
in IoMT frameworks, IEEE Trans. on Consumer Electronics 66 (2020) pp. 327-335.

DOI: $10.1109 /$ TCE.2020.3011966

[73] Z. A. Al-Odat, S. K. Srinivasan, E. M. Al-Qtiemat, S. Shuja, A reliable IoT-based embedded health care system for diabetic patients, Int. J. on Advances in Internet Technology (2019) pp 5060. Online [Accessed 28 June 2021]

http://www.iariajournals.org/internet technology/inttech v12 n12 2019 paged.pdf

[74] B. Karaböce, Challenges for medical metrology, IEEE Instrumentation \& Measurement Magazine 23 (2020) pp. 48-55. DOI: $\underline{10.1109 / M I M .2020 .9126071}$
[75] E. Balestrieri, S. Rapuano, Instruments and methods for calibration of oscillometric blood pressure measurement devices, IEEE Trans. on Instrumentation and Measurement 59 (2010) pp. 2391-2404.

DOI: $10.1109 /$ TIM.2010.2050978

[76] M. do Céu Ferreira, The role of metrology in the field of medical devices, Int. J. Metrol. Qual. Eng. 2 (2011) pp. 135-140. DOI: $10.1051 /$ ijmqe/2011101 